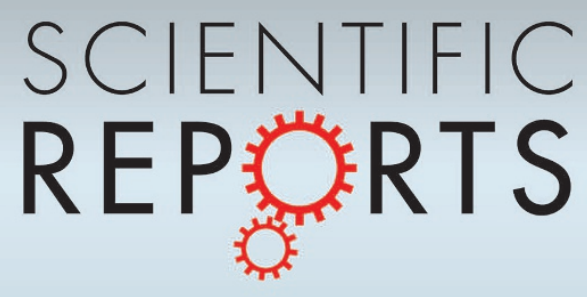

OPEN

SUBJECT AREAS:

ELECTRONIC DEVICES

ELECTRICAL AND ELECTRONIC

ENGINEERING

APPLIED PHYSICS

MAGNETIC DEVICES

Received

4 February 2013

Accepted

8 October 2013

Published

24 October 2013

Correspondence and requests for materials should be addressed to

K.R. (royk@vcu.edu)

\section{Binary switching in a 'symmetric' potential landscape}

\author{
Kuntal Roy', Supriyo Bandyopadhyay' \& Jayasimha Atulasimha ${ }^{2}$
}

'Department of Electrical and Computer Engineering, Virginia Commonwealth University, Richmond, VA 23284, USA,
${ }^{2}$ Department of Mechanical and Nuclear Engineering, Virginia Commonwealth University, Richmond, VA 23284, USA.

A binary switch is the basic building block for information processing. The potential energy profile of a bistable binary switch is a 'symmetric' double well. The traditional method of switching it from one state (one well) to the other is to tilt the profile towards the desired state. Here, we present a case, where no such tilting is necessary to switch successfully, even in the presence of thermal noise. This happens because of the built-in dynamics inside the switch itself. It differs from the general perception on binary switching that in a 'symmetric' potential landscape, the switching probability is $\mathbf{5 0 \%}$ in the presence of thermal noise. Our results, considering the complete three-dimensional potential landscape, demonstrate intriguing phenomena on binary switching mechanism. With experimentally feasible parameters, we theoretically demonstrate such intriguing possibility in electric field induced magnetization switching of a shape-anisotropic single-domain magnetostrictive nanomagnet with two stable states at room-temperature.

U nderstanding the operation of a binary switch, i.e., how switching takes place from one stable state to another, enables us to design a better switch to meet our ever-increasing demand to store, process, and communicate information. From around mid-nineties, the methodology of basic binary switching for information processing as conceived by famous scientist Landauer and others is based on the fact that an externally introduced tilt or asymmetry in the potential landscape of a bistable element in the desired direction of switching is necessary ${ }^{1-4}$. This can be achieved by utilizing an external magnetic field in an elliptical singledomain nanomagnet with two stable magnetization states along the ellipse's major axis acting as a binary switch ${ }^{4}$. The magnetic field, directed along the desired direction of switching, results in a 'tilt' in the magnet's double-well potential profile and this tilt generates a motion along the direction of switching. As long as the tilt is sufficient to beat the thermal agitations, switching with a tolerable error probability is achieved. Such tilt or asymmetry in potential landscape is usually deemed to be necessary for switching to take place. Fig. 1a depicts such traditional methodology of binary switching.

We point out here that the magnetization switching mechanism using spin-transfer-torque $e^{5,6}$, in which a spinpolarized current is passed through a nanomagnet, is analogous to the traditional methodology. Even if the spintransfer-torque has a component that cannot be absorbed as an effective potential ${ }^{5}$, the direction of the externally applied spin-polarized current creates an equivalent tilt or asymmetry causing the switching in the desired direction. However, magnetization reversal is possible by spin-torque induced precessional motion ${ }^{7,8}$ as well as by the electric field induced magnetic anisotropy ${ }^{9,10}$. There is also mechanism of bidirectional electron pump based on Coulomb blockade in single electron tunneling devices ${ }^{11,12}$.

In this article, we show that no such tilting of potential landscape of the bistable switch by external means is necessary to switch successfully, even in the presence of thermal noise. Fig. $1 \mathrm{~b}$ illustrates the basic concept underlying the proposed methodology of binary switching. We consider the complete three-dimensional potential landscape and demonstrate that the internal dynamics can provide an equivalent asymmetry without any requirement of making the potential landscape asymmetric. Note that for both the cases (traditional and proposed methodologies), it is necessary to lower the energy barrier and make the monostable well deep enough to withstand thermal fluctuations. For a magnetostrictive nanomagnet on which our study is based, stress is the external agent inverting the potential landscape. The stress can be generated on a magnetostrictive nanomagnet by applying a voltage on a piezoelectric layer elastically coupled to it, i.e., using 2-phase multiferroic composites $^{13-28}$. This can potentially be the basis of ultra-low-energy computing for our future information processing paradigm. 
a Traditional methodology
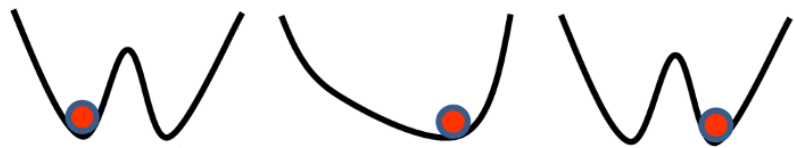

b Proposed methodology
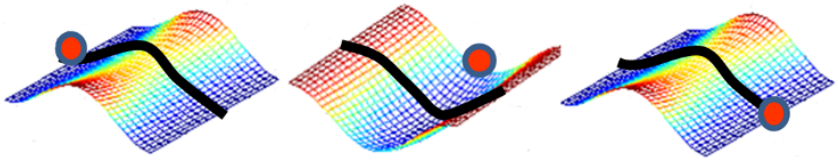

Figure 1 Traditional methodology versus the proposed methodology of binary switching. (a) In traditional methodology of binary switching, the potential landscape is tilted towards the direction of switching alongwith the lowering of energy barrier separating the two stable states. Note that there are two external agents involved, one makes the potential landscape monostable and the other one that tilts the potential landscape. At the end, the potential profile is restored back to that of initial stage to complete the switching process. (b) In the proposed methodology of binary switching, the potential profile remains always 'symmetric', i.e., energy barrier is lowered but the potential landscape is not tilted to favor the final state. Switching occurs due to internal dynamics considering complete threedimensional potential landscape and full three-dimensional motion. Note that in this case only one external agent is involved since it does not require tilting the potential landscape. For a nanomagnet acting as a binary switch, the bold line in its potential landscape corresponds to when magnetization resides on the magnet's plane. Deflection of magnetization from the magnet's plane corresponds to out-of-plane excursion of magnetization, which is the physical mechanism of switching in this case, as we demonstrate in this article.

\section{Results}

Modeling. Switching dynamics of a single-domain poly-crystalline magnetostrictive particle in the presence of thermal fluctuations is modeled using the stochastic Landau-Lifshitz-Gilbert (LLG) equation $^{29-31}$ that describes the time-evolution of the magnetization under various torques. There are three torques to consider here: torque due to shape anisotropy of the nanomagnet, torque due to generated stress anisotropy, and the torque associated with random thermal fluctuations. We consider a magnetostrictive singledomain $^{32}$ nanomagnet made of polycrystalline Terfenol-D that is shaped like an elliptical cylinder. Fig. 2a shows the magnetization in three-dimensional space. We will call the $z$-axis the easy axis, the $y$-axis the in-plane hard axis, and the $x$-axis the out-of-plane hard axis based on the chosen dimensions of the nanomagnet. The two stable magnetization states are along the $\pm z$-axis. In order to switch the magnetization, uniaxial compressive stress is applied in the $z$-direction.

In the macrospin approximation ${ }^{32-37}$, the magnetization $\mathbf{M}$ of the nanomagnet has a constant magnitude but a variable direction, so that we can represent it by a vector of unit norm $\mathbf{n}_{\mathbf{m}}=\mathbf{M} / M=\hat{\mathbf{e}}_{\mathrm{r}}$ where $\hat{\mathbf{e}}_{\mathrm{r}}$ is the unit vector in the radial direction in spherical coordinate system represented by $(r, \theta, \phi), \theta\left[\theta \in\left(0^{\circ}, 180^{\circ}\right)\right]$ is the polar angle, and $\phi\left[\phi \in\left(0^{\circ}, 360^{\circ}\right)\right]$ is the azimuthal angle. Magnetization resides on the magnet's plane if $\phi= \pm 90^{\circ}(y-z$ plane $)$. The unit vectors associated with $\theta$ and $\phi$ rotations are $\hat{\mathbf{e}}_{\theta}$ and $\hat{\mathbf{e}}_{\phi}$, respectively.

The total potential energy of the stressed nanomagnet is the sum of the shape anisotropy energy and stress anisotropy energy ${ }^{36}$ given by

$$
E=B(\phi) \sin ^{2} \theta
$$

a
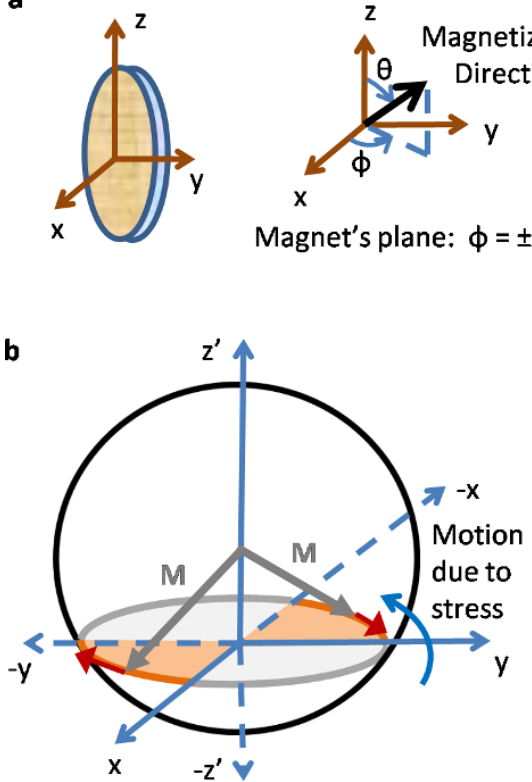

c

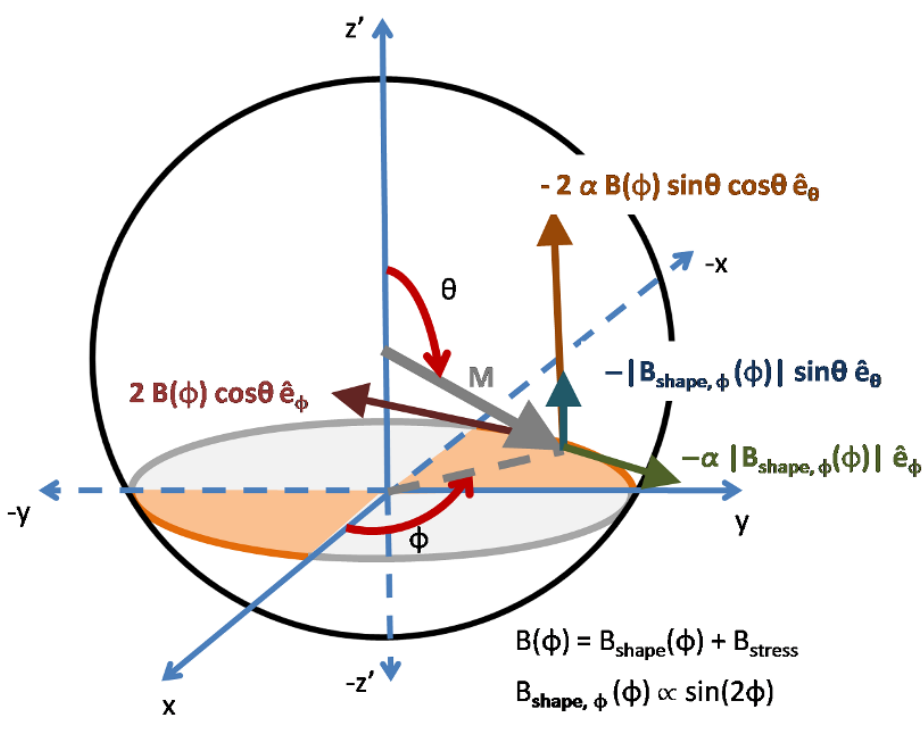

Figure $2 \mid$ Illustration of magnetization's motion in three-dimensional space. (a) Cross section of the nanomagnet and axes assignment. (b) The applied stress tries to lift the magnetization $\mathbf{M}$ out of the magnet's plane while the $\hat{\mathbf{e}}_{\phi}$-component of the shape anisotropy torque due to Gilbert damping ${ }^{29,30}$ tries to bring it back to the plane $\left(\phi= \pm 90^{\circ}\right)$. This stabilizes the value of $\phi$, but it happens only in the "good" quadrants $\phi \in\left(90^{\circ}, 180^{\circ}\right)$ and $\phi \in\left(270^{\circ}, 360^{\circ}\right)$. For the other two quadrants $\phi \in\left(0^{\circ}, 90^{\circ}\right)$ and $\phi \in\left(180^{\circ}, 270^{\circ}\right)$, such counteraction does not happen and we term them as "bad" quadrants. (c) Illustration of the motion of magnetization $\mathbf{M}$ in three-dimensional space under various torques generated due to shape and stress anisotropy alongwith considering the damping of magnetization ( $\alpha$ is the phenomenological damping parameter $\left.{ }^{29,30}\right)$. Note that the dependence of shape anisotropy energy on $\phi$ has generated two additional motions $-\left|B_{\text {shape }, \phi}(\phi)\right| \sin \theta \hat{\mathbf{e}}_{\theta}$ and $-\alpha\left|B_{\text {shape, } \phi}(\phi)\right| \theta \hat{\mathbf{e}}_{\phi}$. (See text for details.) The quadrant $\phi \in\left(90^{\circ}, 180^{\circ}\right)$ is chosen for illustration; choice of the other "good" quadrant $\phi \in\left(270^{\circ}, 360^{\circ}\right)$ is analogous. 
where

$$
B(\phi)=B_{\text {shape }}(\phi)+B_{\text {stress }},
$$

$B_{\text {shape }}(\phi)$ is the $\phi$-dependent strength of shape anisotropy energy ( $\propto$ $\cos ^{2} \phi+$ constant term), which is minimum on the magnet's plane $\phi$ $= \pm 90^{\circ}$, and $B_{\text {stress }}$ is the strength of the stress anisotropy energy. (Details are provided in Supplementary Section S1.)

The torque due to shape and stress anisotropy is derived from the gradient of potential profile as

$$
\begin{aligned}
\mathbf{T}_{\mathbf{E}} & =-\mathbf{n}_{\mathbf{m}} \times \nabla E \\
& =-\hat{\mathbf{e}}_{\mathbf{r}} \times\left(\frac{\partial E}{\partial \theta} \hat{\mathbf{e}}_{\theta}+\frac{1}{\sin \theta} \frac{\partial E}{\partial \phi} \hat{\mathbf{e}}_{\phi}\right) \\
& =-2 B(\phi) \sin \theta \cos \theta \hat{\mathbf{e}}_{\phi}-B_{\text {shape }, \phi}(\phi) \sin \theta \hat{\mathbf{e}}_{\theta}
\end{aligned}
$$

where $B_{\text {shape, } \phi}(\phi) \propto \sin (2 \phi)$.

The torque due to thermal fluctuations is treated via a random magnetic field $\mathbf{h}$ expressed as

$$
\mathbf{h}=h_{x} \hat{\mathbf{e}}_{\mathbf{x}}+h_{y} \hat{\mathbf{e}}_{\mathbf{y}}+h_{z} \hat{\mathbf{e}}_{\mathbf{z}}
$$

where $h_{x}, h_{y}$, and $h_{z}$ are the three components of $\mathbf{h}$ in $x$-, $y$-, and $z$ direction, respectively in Cartesian coordinates. We will assume the properties of the random field, $\mathbf{h}$ as described in Ref. 31 .

The random thermal field can be expressed as ${ }^{31,37,38}$

$$
h_{i}=\sqrt{\frac{2 \alpha k T}{|\gamma|\left(1+\alpha^{2}\right) M \Delta t}} G_{0,1} \quad(i=x, y, z)
$$

where $\alpha$ is the phenomenological Gilbert damping parameter, $\gamma$ is the gyromagnetic ratio of electrons, $1 / \Delta t$ is proportional to the attempt frequency of the random thermal field, $k$ is the Boltzmann constant, $\Delta t$ is the simulation time-step, $T$ is temperature, and $G_{(0,1)}$ is a Gaussian distribution with zero mean and unit standard deviation ${ }^{39}$. The simulation time-step $\Delta t$ should be selected small enough so that decreasing that step further does not make any significant difference in the results. Accordingly, the thermal torque can be written as

$$
\mathbf{T}_{\mathbf{T H}}=M \mathbf{n}_{\mathbf{m}} \times \mathbf{h}=P_{\theta} \hat{\mathbf{e}}_{\phi}-P_{\phi} \hat{\mathbf{e}}_{\theta}
$$

where

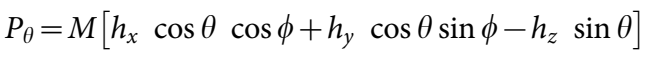

$$
\begin{aligned}
& P_{\phi}=M\left[\begin{array}{lll}
h_{y} & \cos \phi-h_{x} & \sin \phi
\end{array}\right] .
\end{aligned}
$$

Additionally, there is motion due to Gilbert damping ${ }^{29,30}$ (perpendicular to precessional motion) through which magnetization relaxes towards the minimum energy position in the magnet's potential landscape. The magnetization dynamics under the action of these two torques $\mathrm{T}_{\mathrm{E}}$ and $\mathrm{T}_{\mathrm{TH}}$ is described by the stochastic LandauLifshitz-Gilbert (LLG) equation as follows.

$$
\frac{d \mathbf{n}_{\mathbf{m}}}{d t}-\alpha\left(\mathbf{n}_{\mathbf{m}} \times \frac{d \mathbf{n}_{\mathbf{m}}}{d t}\right)=-\frac{|\gamma|}{M}\left[\mathbf{T}_{\mathbf{E}}+\mathbf{T}_{\mathbf{T H}}\right]
$$

where $\alpha$ is the phenomenological Gilbert damping parameter and $\gamma$ is the gyromagnetic ratio of electrons. Solving the above equation, we get the following coupled equations for the dynamics of $\theta$ and $\phi$ (see Supplementary Section S1 for detailed steps)

$$
\begin{aligned}
\left(1+\alpha^{2}\right) \frac{d \theta}{d t}= & \frac{|\gamma|}{M}\left[B_{\text {shape }, \phi}(\phi) \sin \theta-\right. \\
& \left.2 \alpha B(\phi) \sin \theta \cos \theta+\left(\alpha P_{\theta}+P_{\phi}\right)\right]
\end{aligned}
$$

$$
\begin{aligned}
\left(1+\alpha^{2}\right) \frac{d \phi}{d t}= & \frac{|\gamma|}{M}\left[\alpha B_{\text {shape }, \phi}(\phi)+2 B(\phi) \cos \theta-\right. \\
& \left.\{\sin \theta\}^{-1}\left(P_{\theta}-\alpha P_{\phi}\right)\right] \quad(\sin \theta \neq 0) .
\end{aligned}
$$

We will ignore the random thermal torque while explaining the basic magnetization dynamics, however, we will discuss the consequences of room-temperature thermal fluctuations with simulation results later. We will assume that the magnetization starts from $\theta \simeq 180^{\circ}$ $(-z$-axis $)$ and the applied stress tries to switch it to $\theta \simeq 0^{\circ}(+z$-axis $)$.

Out-of-plane excursion of magnetization. First, we will intuitively describe how magnetization is deflected from its plane $\left(\phi= \pm 90^{\circ}\right.$, i.e., $y$ - $z$ plane), and gets stabilized out-of-plane as depicted in Fig. $2 b$. Dependence of shape-anisotropy energy on azimuthal angle $\phi$ (rather than assuming $\phi= \pm 90^{\circ}$ ) generates additional motions of magnetization in $\hat{\mathbf{e}}_{\theta}$ and $\hat{\mathbf{e}}_{\phi}$ directions (see the motions containing the term $B_{\text {shape, } \phi}(\phi)$ in Fig. $2 c$ and equations (10) and (11)); both the components are proportional to $\sin (2 \phi)$ and vanish when $\phi=$ $\pm 90^{\circ}$, but additionally the $\phi$-component is proportional to the damping parameter $\alpha$. As shown in the Fig. $2 b$, the applied stress produces a torque that tries to rotate the magnetization anticlockwise and forces it to reside out of magnet's plane. As magnetization is deflected from the plane of the magnet $\left(\phi= \pm 90^{\circ}\right)$, the $\phi$ component of the additional torque due to shape anisotropy as mentioned above $[\propto \alpha \sin (2 \phi)]$ would try to bring the magnetization back to its plane. Because of such counteraction, out of the four quadrants for $\phi$, the magnetization would be stable in the second or the fourth quadrant [i.e., $\left(90^{\circ}, 180^{\circ}\right)$ or $\left.\left(270^{\circ}, 360^{\circ}\right)\right]$. Note that $\sin (2 \phi)$ is negative in these two quadrants counteracting the precessional motion due to stress. We would call these two quadrants "good" quadrants and the other two (first and third) quadrants as "bad" quadrants, the reasoning behind which would be more transparent onwards. Consideration of the torques due to $\phi$ dependence of shape anisotropy energy is central to the dynamics we present in this article. The important lesson to take away is that $\phi$ is stable only in "good" quadrants, which is why $\phi$ is much more likely to be in a "good" quadrant during switching.

Magnetization's motion in three-dimensional space. We will now describe the motion of magnetization intuitively under various torques originating from shape and stress anisotropy as shown in Fig. 2c. We intend the motion of magnetization to be along the $-\hat{\mathbf{e}}_{\theta}$ direction since we are switching magnetization from $\theta \simeq 180^{\circ}$ towards $\theta \simeq 0^{\circ}$. The precessional motion of magnetization due to torque generated by the applied stress is in the $+\hat{\mathbf{e}}_{\phi}$ direction, but the damping of magnetization generates an additional motion, which is perpendicular to both the direction of magnetization $\left(\hat{\mathbf{e}}_{r}\right)$ and $+\hat{\mathbf{e}}_{\phi}$, i.e., in $-\hat{\mathbf{e}}_{\theta}$ direction. These two motions are depicted as $2 B(\phi) \cos \theta \hat{\mathbf{e}}_{\phi}$ and $-2 \alpha B(\phi) \sin \theta \cos \theta \hat{\mathbf{e}}_{\theta}$, respectively in Fig. $2 c$, where $\alpha$ is the damping parameter and the quantity $B(\phi)$ includes a term due to shape anisotropy $B_{\text {shape }}(\phi)$ in addition to the stress anisotropy term $B_{\text {stress. }}$. The quantity $B_{\text {stress }}$ is negative and it must beat the shape anisotropy term $B_{\text {shape }}(\phi)$ for switching to get started. Mathematically, note that both the quantities $B(\phi)$ and $\cos \theta$ are negative in the interval $180^{\circ} \geq \theta \geq 90^{\circ}$. Hence, magnetization switches towards its desired direction due to the applied stress. However, this damped motion in $-\hat{\mathbf{e}}_{\theta}$ direction is weak because of the multiplicative factor $\alpha$, which is usually much less than one (e.g., $\alpha$ $=0.1$ for Terfenol-D).

As magnetization rotates out-of-plane due to applied stress, and stays in a "good" quadrant for $\phi$ [i.e., $\left(90^{\circ}, 180^{\circ}\right)$ or $\left(270^{\circ}, 360^{\circ}\right)$ ] as described earlier (Fig. 2b), it generates a motion of magnetization in the $-\hat{\mathbf{e}}_{\theta}$ direction due to $\phi$-dependence of shape anisotropy energy. 
Subsequently, a damped motion is generated too in the $-\hat{\mathbf{e}}_{\phi}$ direction. These two motions are depicted as $-\left|B_{\text {shape, } \phi}(\phi)\right| \sin \theta \hat{\mathbf{e}}_{\theta}$ and $-\alpha\left|B_{\text {shape, } \phi}(\phi)\right| \hat{\mathbf{e}}_{\phi}$, respectively in Fig. $2 c$, where $B_{\text {shape, } \phi}(\phi) \propto$ $\sin (2 \phi)$. Note that in the "good" quadrants for $\phi, B_{\text {shape, } \phi}(\phi)$ is negative. Thus, keeping the magnetization out of magnet's plane in a "good" quadrant is beneficial in switching the magnetization in its desired direction. In fact, it can eventually increase the switching speed by a couple of orders of magnitude. In case the magnetization resides out-of-plane but in a "bad" quadrant, it would have resisted the motion of magnetization in its desired direction of switching. A higher magnitude of stress keeps the magnetization out of magnet's plane, but more inside a "good" quadrant counteracting the random thermal kicks possibly acting in the opposite direction, however, the damped motion $-\alpha\left|B_{\text {shape, } \phi}(\phi)\right| \hat{\mathbf{e}}_{\phi}$ tries to bring the magnetization back towards the magnet's plane. As these two motions counteract each other (Fig. 2c), the magnetization keeps moving in the $-\hat{\mathbf{e}}_{\theta}$ direction and eventually reaches the $x-y$ plane $\left(\theta=90^{\circ}\right)$. Note that without damping, such counteraction does not happen and magnetization precesses alternatingly through "good" and "bad" quadrants.

Upon reaching at $\theta=90^{\circ}$, if magnetization stays in a "good" quadrant for $\phi$ [i.e., $\left(90^{\circ}, 180^{\circ}\right)$ or $\left(270^{\circ}, 360^{\circ}\right)$ ], the torque on the magnetization is in the correct direction so that it can traverse towards $\theta \simeq 0^{\circ}$ (Figs. $3 \mathrm{a}$ and $3 \mathrm{~b}$ ). This once again justifies of termino$\operatorname{logy}$ ("good" or "bad") used for the four quadrants of $\phi$. Note that at $\theta=90^{\circ}$ (i.e., $\cos \theta=0$ ), the effect of stress on the magnetization rotation has diminished completely. The only two motions that are active at that point are $-\left|B_{\text {shape, } \phi}(\phi)\right| \sin \theta \hat{\mathbf{e}}_{\theta}$ and $-\alpha\left|B_{\text {shape, } \phi}(\phi)\right| \hat{\mathbf{e}}_{\phi}$ (Fig. 2c). Since $\alpha \ll 1$, magnetization quickly gets out of the $\theta=90^{\circ}$ position and as the magnetization vector gets deflected from $\theta=90^{\circ}$ towards $\theta=0^{\circ}$, the effect of stress comes into play.

Stress cycle, magnetization directions, and potential profiles. The stress-cycle alongwith the energy profiles and magnetization directions at different instants of time during switching of magnetization is shown in the Fig. 4 . At time $t_{0}$, the magnetization direction is along the easy axis $\theta \simeq 180^{\circ}$ and the potential landscape of the nanomagnet is unperturbed by stress. The potential profile of the magnet is 'symmetric' in both $\theta$ - and $\phi$-space with two degenerate minima at $\theta=0^{\circ}, 180^{\circ}$ and a maximum at $\theta=90^{\circ}$ in $\theta$-space. The anisotropy in the barrier is due to shape anisotropy only, which is $\sim 44 \mathrm{kT}$ at room-temperature using the magnet's dimensions and material parameters for Terfenol-D (Supplementary Table S1, Refs. 40-44). Note that the barrier height separating the two stable states $(\theta$ $=0^{\circ}$ and $\left.180^{\circ}\right)$ is meant when the magnetization resides in-plane (i.e., $\phi= \pm 90^{\circ}$ ) of the magnet. The barrier goes higher when the magnetization is deflected from $\phi=90^{\circ}$ as shown in Fig. $4 \mathrm{c}$ (at time $t_{0}$ ). The barrier is highest when the magnetization points along the out-of-plane direction $\left(\phi=0^{\circ}\right.$ or $\left.180^{\circ}\right)$, which is due to the small thickness of the nanomagnet compared to the lateral dimensions. Magnetization can start from any angle $\phi_{\text {initial }} \in\left(0^{\circ}, 360^{\circ}\right)$ in the presence of thermal noise (Supplementary Fig. S2).

As a compressive stress is ramped up on the nanomagnet between time instants $t_{0}$ and $t_{1}$, the potential landscape in $\theta$-space becomes monostable near $\phi= \pm 90^{\circ}$ provided a sufficient stress is applied. The potential barrier near $\phi=0^{\circ}$ or $180^{\circ}$ may not become monostable in $\theta$-space since the barrier height is high therein, however, that is not necessary for switching. Since application of stress rotates the magnetization in $\phi$-direction, it can eventually come near $\phi=$ $\pm 90^{\circ}$ and starts switching from $\theta \simeq 180^{\circ}$ towards $\theta=90^{\circ}$ (Supplementary Fig. S3). The minimum energy position between time instants $t_{1}$ and $t_{2}$ is at $\left(\theta=90^{\circ}, \phi= \pm 90^{\circ}\right)$. From Fig. $4 \mathrm{c}$, we can see that the potential profile at time instant $t_{1}$ is still 'symmetric'.

Stress is held constant between time instants $t_{1}$ and $t_{2}$, and the magnetization eventually reaches the $x-y$ plane $\left(\theta=90^{\circ}\right)$. For a fast enough ramp rate and high enough stress, magnetization will reside in a "good" quadrant as explained before. This will dictate that magnetization traverses in the correct direction towards $\theta=0^{\circ}$. A fast enough ramp rate will facilitate that the magnetization does not backtrack even after crossing $\theta=90^{\circ}$ towards $\theta=0^{\circ}$. If stress is held constant for a longer time, the magnetization would have higher tendency to collapse on the magnet's plane following which thermal fluctuations may scuttle the magnetization either in a "good" quadrant or in a "bad" quadrant, making the success rate $50 \%$.

Note that we reverse the stress (compression to tensile) between time instants $t_{2}$ and $t_{4}$ rather than just withdrawing it, which makes the potential landscape of the nanomagnet more steep in $\theta$-space. But, it does not necessarily mean that the switching will be completed always faster since reversal of stress can cause magnetization to

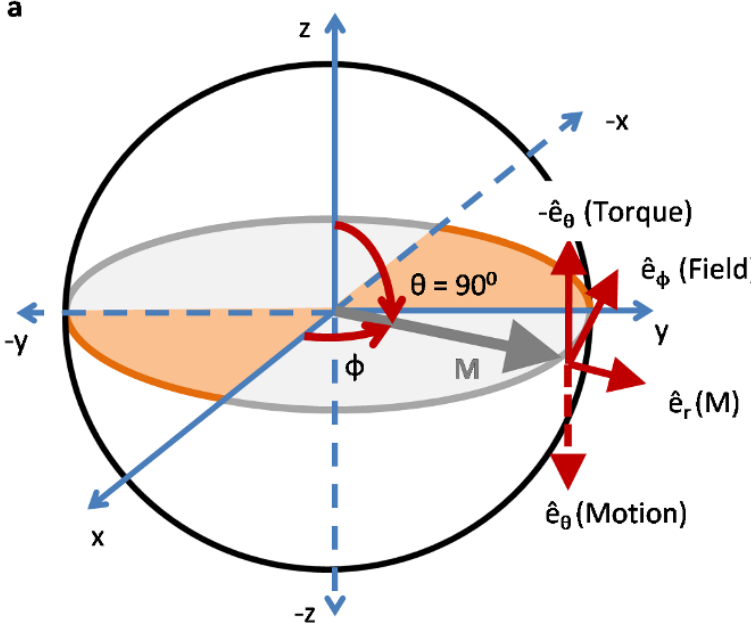

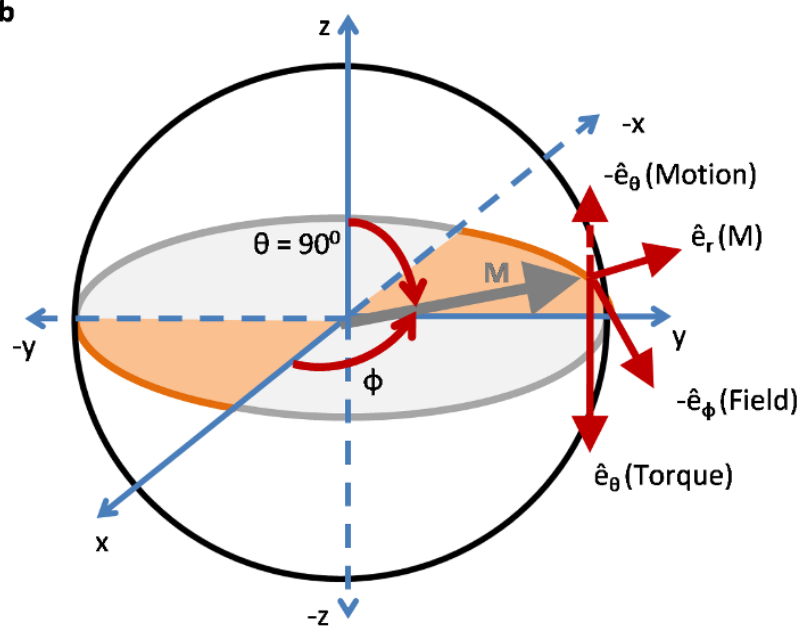

Figure $3 \mid$ Field and torque acting on the magnetization M when it comes on the $\boldsymbol{x}$ - $y$ plane $\left(\boldsymbol{\theta}=\mathbf{9 0 ^ { \circ }}\right)$. (a) $\phi \in\left(0^{\circ}, 90^{\circ}\right)$. The field always tries to keep the magnetization on the magnet's plane $\left(\phi= \pm 90^{\circ}\right)$. For this "bad" quadrant $\phi \in\left(0^{\circ}, 90^{\circ}\right)$, magnetization backtracks towards $\theta \simeq 180^{\circ}$ causing a switching failure. Choice of the other "bad" quadrant $\phi \in\left(180^{\circ}, 270^{\circ}\right)$ is analogous. (b) $\phi \in\left(90^{\circ}, 180^{\circ}\right)$. The field again tries to keep the magnetization on the magnet's plane $\left(\phi= \pm 90^{\circ}\right)$. For this "good" quadrant $\phi \in\left(90^{\circ}, 180^{\circ}\right)$, magnetization can traverse towards its destination $\theta \simeq 0^{\circ}$. Choice of the other "good" quadrant $\phi \in\left(270^{\circ}, 360^{\circ}\right)$ is analogous. Note that the motion of magnetization is opposite to the direction of torque exerted on it since the Landé $g$-factor for electrons is negative. If magnetization starts from the other easy axis $\theta \simeq 0^{\circ}$ and we switch it towards $\theta \simeq 180^{\circ}$, the roles of the four quadrants of $\phi$ would have been exactly opposite. 
a

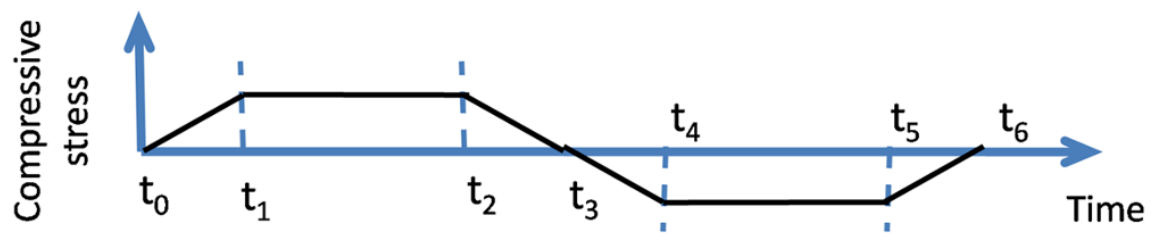

b
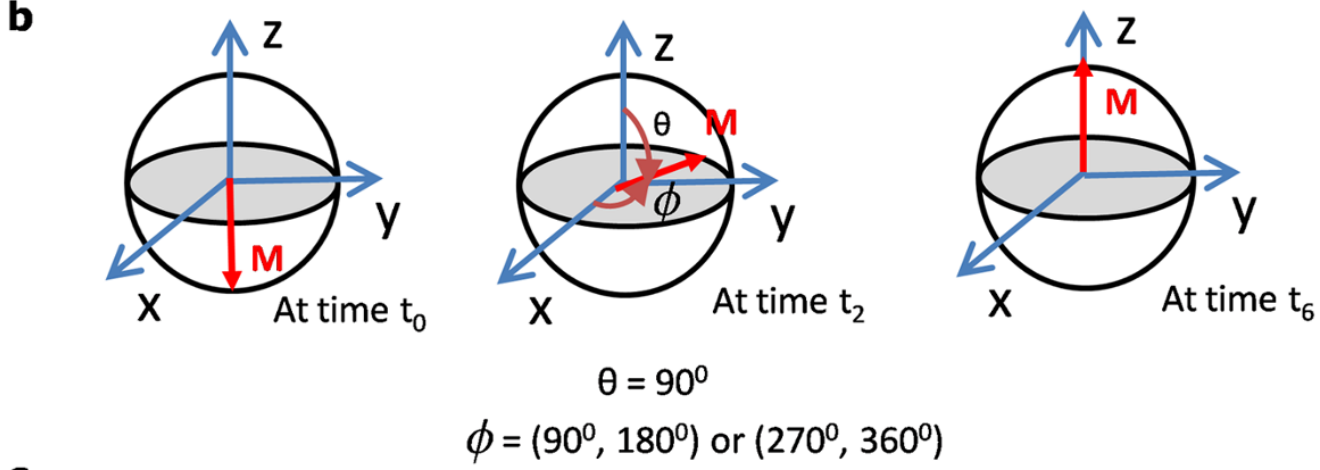

C
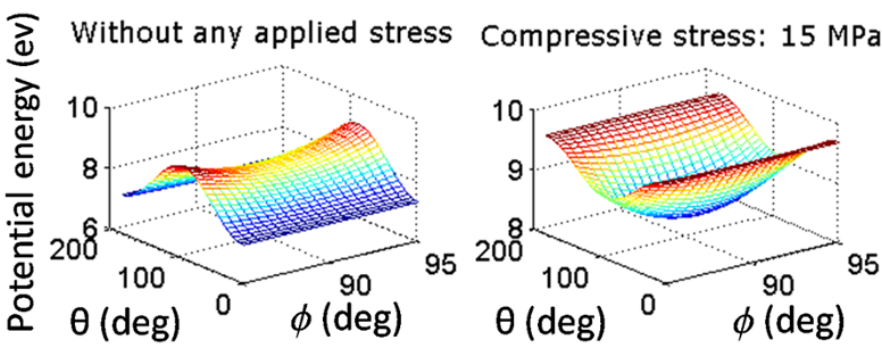

Tensile stress: $15 \mathrm{MPa}$

\section{At times $t_{0}, t_{3}$, and $t_{6} \quad$ Between times $t_{1}$ and $t_{2}$}

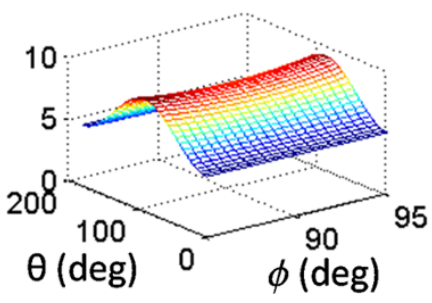

Between times $t_{4}$ and $t_{5}$

Figure 4 Stress cycle, magnetization directions, and potential profiles at different time instants during switching of magnetization. (a) Stress-cycle on the magnetostrictive nanomagnet. (b) Magnetization directions at different instants of time. (c) Potential landscapes of the magnetostrictive nanomagnet in relaxed, compressively stressed, and expansively stressed conditions. Note that the three-dimensional potential landscape has never been made asymmetric to favor the final state during switching.

traverse into "bad" quadrants in $\phi$-space causing magnetization to precess and switching delay may be eventually increased. Such increase in switching delay may happen particularly for higher stress levels. However, reversing the stress makes the success rate of switching a bit $(<5 \%)$ higher in the presence of thermal fluctuations. The tensile stress is held constant and when $\theta$ becomes $\leq 5^{\circ}$, switching is deemed to have completed. The stress is withdrawn at the end between time instants $t_{5}$ and $t_{6}$ to complete the switching process.

Magnetization dynamics. Fig. 5 plots the magnetization dynamics for different values of $\phi_{\text {initial }}$ (while keeping fixed values of $\theta_{\text {initial }}$, stress, and ramp rate) to depict the significance of out-of-plane excursion of magnetization. For Figs. $5 \mathrm{a}$ and $5 \mathrm{~b}$, the magnetization initially lies in the plane of the magnet $\left(\phi_{\text {initial }}= \pm 90^{\circ}\right)$ and precessional motion of magnetization due to applied stress is in the $+\hat{\mathbf{e}}_{\phi}$ direction, which increases $\phi$ with time. Thus, the magnetization starts out in the "good" quadrant. Note that both the motions of magnetization (damped motion due to applied stress and the motion due to out-of-plane excursion depicted as $-2 \alpha B(\phi) \sin \theta \cos \theta \hat{\mathbf{e}}_{\theta}$ and $-\left|B_{\text {shape, } \phi}(\phi)\right| \sin \theta \hat{\mathbf{e}}_{\theta}$, respectively in the Fig. $2 \mathrm{c})$ are in the $-\hat{\mathbf{e}}_{\theta}$ direction so $\theta$ decreases with time and the magnetization rotates in the correct direction towards $\theta=90^{\circ}$. The increasing out-of-plane excursion of the magnetization vector due to $\phi$ increasing with time however is opposed by the damped motion due to out-of-plane excursion (depicted as $-\alpha\left|B_{\text {shape, } \phi}(\phi)\right| \hat{\mathbf{e}}_{\phi}$ in Fig. 2c), which tries to bring the magnetization back to the magnet's plane. These two effects quickly balance and $\phi$ assumes a stable value in the "good" quadrant as seen in the plots (the flat regions of the $\phi$-plots). When $\theta$ reaches $90^{\circ}$, the torque due to stress and shape anisotropy vanishes. At this point, we start to reverse the stress and the damped motion due to stress and shape anisotropy eventually becomes again in the $-\hat{\mathbf{e}}_{\theta}$ direction. That continues to rotate the magnetization in the right direction towards $\theta=0^{\circ}$, ending in successful switching. Slightly past $0.4 \mathrm{~ns}$, continuing $\phi$-rotation because of precessional motion due to stress and shape anisotropy pushes $\phi$ into a "bad" quadrant, but eventually it escapes into the other "good" quadrant. This brief excursion into the "bad" quadrant causes the ripple in $\theta$-plots.

For Figs. $5 \mathrm{c}$ and $5 \mathrm{~d}$, the magnetization vector is initially lifted far out of the magnet's plane $\left(\phi_{\text {initial }}=0^{\circ}, 180^{\circ}\right)$, where the huge out-ofplane shape anisotropy cannot be overcome by the stress anisotropy and $B(\phi)$ becomes positive, i.e., $\left|B_{\text {stress }}\right|<\left|B_{\text {shape }}(\phi)\right|$. So magnetization precesses in the clockwise direction $\left(-\hat{\mathbf{e}}_{\phi}\right)$ rather than in the anticlockwise direction $\left(+\hat{\mathbf{e}}_{\phi}\right)$. Thus $\phi$ decreases with time, which immediately takes magnetization inside a "good" quadrant and eventually $\left|B_{\text {stress }}\right|$ becomes greater than $\left|B_{\text {shape }}(\phi)\right|$. Then $\phi$ assumes a stable value because of the damped motion due to out-of-plane excursion (depicted as $-\alpha\left|B_{\text {shape, } \phi}(\phi)\right| \hat{\mathbf{e}}_{\phi}$ in Fig. 2c). Afterwards, switching occurs similarly for the cases as in Figs. $5 \mathrm{a}$ and $5 \mathrm{~b}$. Slightly past $0.3 \mathrm{~ns}$, continuing $\phi$ rotation pushes $\phi$ into a "bad" quadrant, but eventually it escapes into the other good quadrant. 


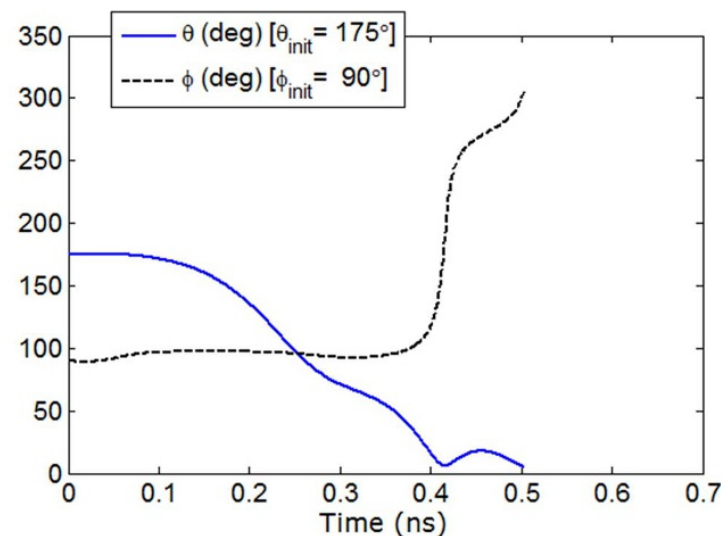

C

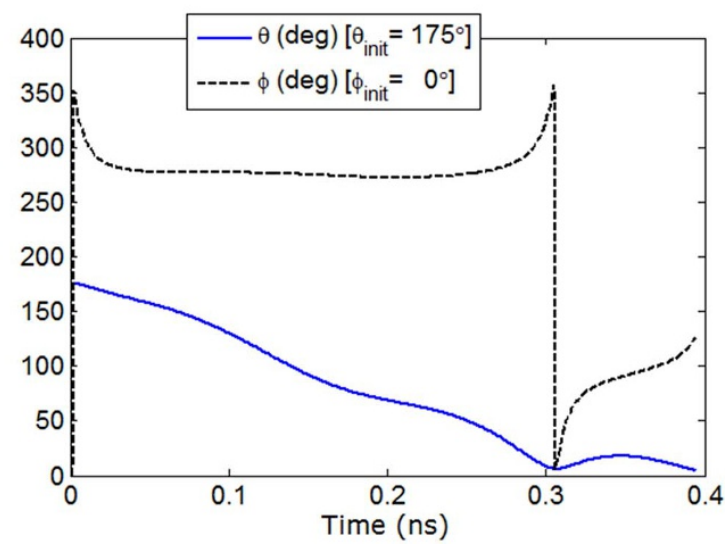

b

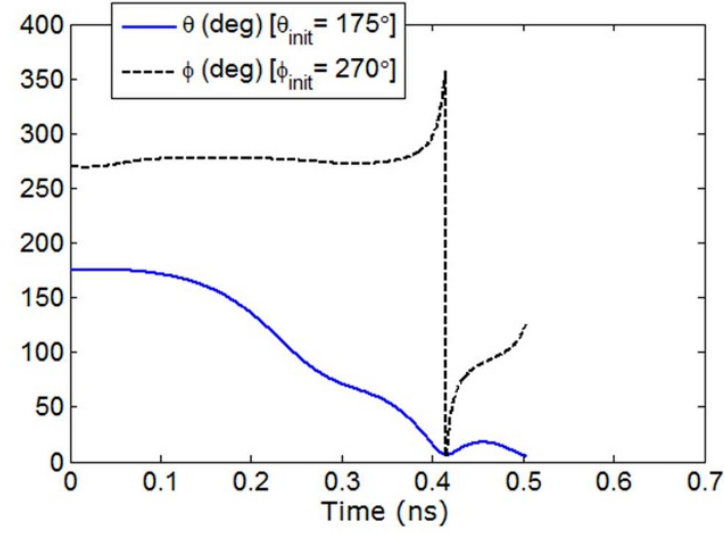

d

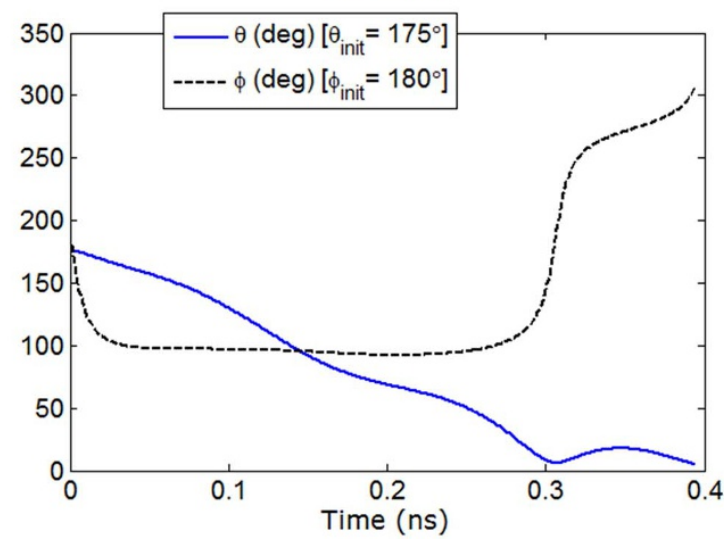

Figure $5 \mid$ Temporal evolution of polar angle $\theta$ and azimuthal angle $\phi$ for a fixed $\theta_{\text {initial }}=175^{\circ}$ and four different values of $\phi_{\text {initial }}=\left\{90^{\circ}, 270^{\circ}, 0^{\circ}\right.$, $\left.180^{\circ}\right\}$. The applied stress is $15 \mathrm{MPa}$ and the ramp duration is $60 \mathrm{ps}$. Thermal fluctuations have been ignored. (a) $\phi_{\text {initial }}=90^{\circ}$. (b) $\phi_{\text {initial }}=270^{\circ}$. (c) $\phi_{\text {initial }}$ $=0^{\circ}$. (d) $\phi_{\text {initial }}=180^{\circ}$. Note that when $\theta$ reaches $90^{\circ}$ or even earlier, $\phi$ always resides in a "good" quadrant $\left[\left(90^{\circ}, 180^{\circ}\right)\right.$ or $\left.\left(270^{\circ}, 360^{\circ}\right)\right]$, which makes the switching successful.

Once again, this brief excursion into the bad quadrant causes the ripple in $\theta$-plots and switching is completed successfully at the end.

Switching failures. Fig. 6 demonstrates a couple of examples when switching fails. In Fig. 6a, when the polar angle $\theta$ reaches $90^{\circ}$, the azimuthal angle $\phi$ has ventured into the "bad" quadrant $\left(0^{\circ}, 90^{\circ}\right)$. Thus, switching eventually fails. In Fig. $6 \mathrm{~b}$, when the polar angle $\theta$ reaches $90^{\circ}$, the azimuthal angle $\phi$ is greater than $90^{\circ}$ and hence is in a "good" quadrant. However, after reaching $\theta \simeq 50^{\circ}$, the magnetization

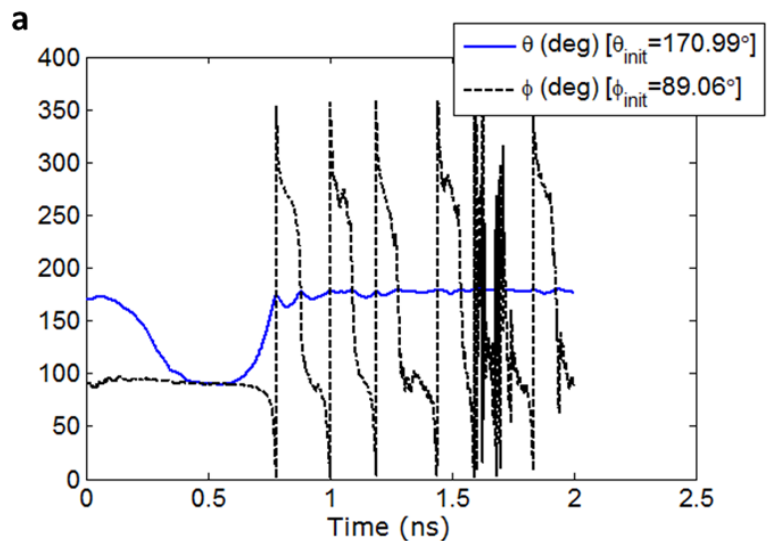

backtracks to the initial state and switching fails. This happens because of the long ramp duration bringing $\phi$ into the "bad" quadrant $\left(0^{\circ}, 90^{\circ}\right)$ and also during the passage of long duration thermal fluctuations have ample opportunity to scuttle the switching. Such switching failure is depicted intuitively in the Fig. 7 and explained below.

As magnetization leaves from $\theta=90^{\circ}$ towards $\theta \simeq 0^{\circ}$ and stress is ramped down, the torque due to stress tries to rotate the azimuthal angle $\phi$ of magnetization clockwise rather than anticlockwise

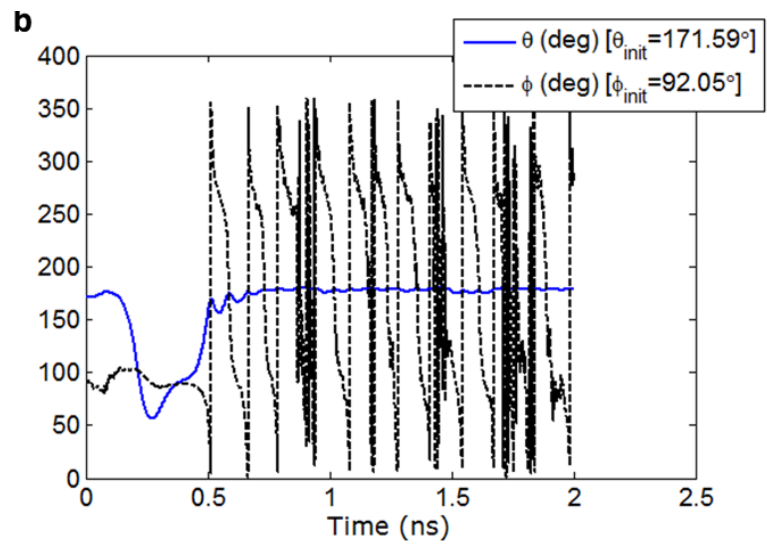

Figure 6 Temporal evolution of the polar angle $\theta$ and azimuthal angle $\phi$ when magnetization fails to switch and backtracks to the initial state. Simulations are carried out at room-temperature $(300 \mathrm{~K})$. (a) The applied stress is $10 \mathrm{MPa}$ and the ramp duration is $60 \mathrm{ps}$. (b) The applied stress is $30 \mathrm{MPa}$ and the ramp duration is $120 \mathrm{ps}$. The ringing in the $\phi$-plots at the end is just due to thermal fluctuations that causes magnetization to roam around $\theta=180^{\circ}$. 


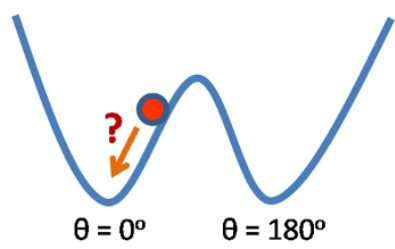

b

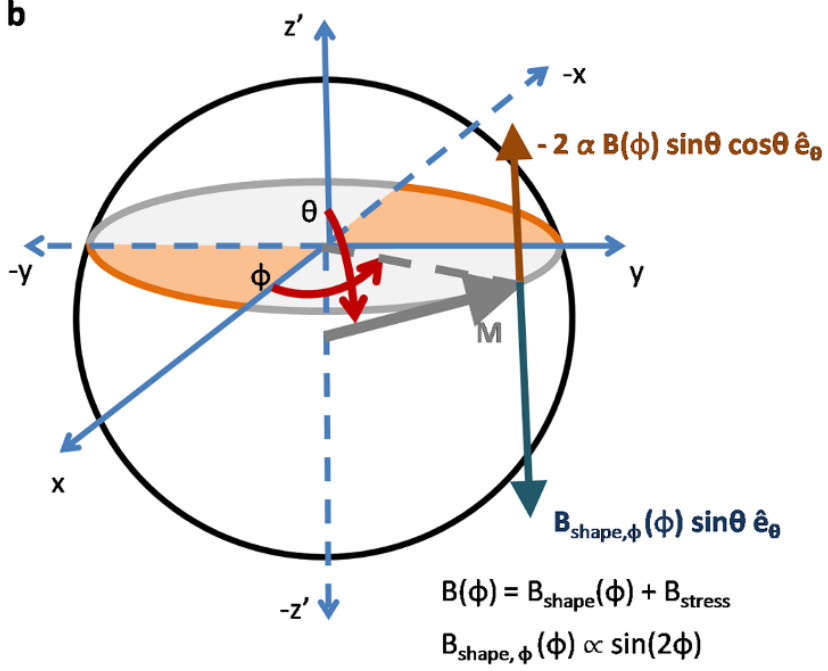

Figure 7 Magnetization can backtrack even after it has crossed the hard axis towards its destination. (a) Magnetization has started from $\theta \simeq 180^{\circ}$ and crossed the hard axis $\theta=90^{\circ}$, but it is well possible that magnetization backtracks towards $\theta \simeq 180^{\circ}$ even without considering the presence of thermal fluctuations. Looking at the two-dimensional magnet's plane and considering two-dimensional motion of magnetization on the magnet's plane, this seems unreasonable in the absence of thermal fluctuations. But, considering the complete three-dimensional potential landscape of the nanomagnet and full three-dimensional motion, this would be plausible even in the absence of thermal fluctuations. (b) Explanation behind magnetization's backtracking even after it has crossed the hard axis towards its destination. Magnetization may switch to the incorrect direction because it is in a "bad" quadrant for $\phi$ and there is a motion of magnetization $B_{\text {shape, } \phi}(\phi) \sin \theta \hat{\mathbf{e}}_{\theta}$ in the unintended direction. The other magnetization's motion $-2 \alpha B(\phi) \sin \theta \cos \theta \hat{\mathbf{e}}_{\theta}$ due to damping is in the intended direction but it may be quite small compared to the other motion and thus magnetization may well backtrack. The quadrant $\phi \in\left(0^{\circ}, 90^{\circ}\right)$ is chosen for illustration; choice of the other "bad" quadrant $\phi \in\left(180^{\circ}\right.$, $\left.270^{\circ}\right)$ is analogous.

(mathematically note that $\cos \theta$ is positive for $90^{\circ} \geq \theta \geq 0^{\circ}$ and $B(\phi)$ is still negative when stress has not been brought down significantly, i.e., still $\left.\left|B_{\text {stress }}\right|>\left|B_{\text {shape }}(\phi)\right|\right)$. For a slow ramp-rate this rotation may be considerable and magnetization can stray into a "bad" quadrant. Moreover, thermal fluctuations can aggravate the scenario. Switching may impede and magnetization vector can backtrack towards $\theta \simeq 180^{\circ}$ causing a switching failure. In this way, switching failure may happen even after the magnetization has crossed the hard axis $\left(\theta=90^{\circ}\right)$ towards its destination $\theta \simeq 0^{\circ}$ (Fig. 7), which is why it does require a fast enough ramp rate during the ramp-down phase of stress. This is an intriguing phenomenon, which comes out after analyzing the magnetization dynamics in a complete three dimensional potential landscape.

Successful switching rates with respect to stress and ramp rate. Fig. 8 plots the successful switching rates at room-temperature $(300 \mathrm{~K})$ when the magnetostrictive nanomagnet is subjected to stress between $10 \mathrm{MPa}$ and $30 \mathrm{MPa}$ with ramp duration (60 ps, $90 \mathrm{ps}$, and $120 \mathrm{ps}$ ) as a parameter. A moderately large number $(10,000)$ of simulations

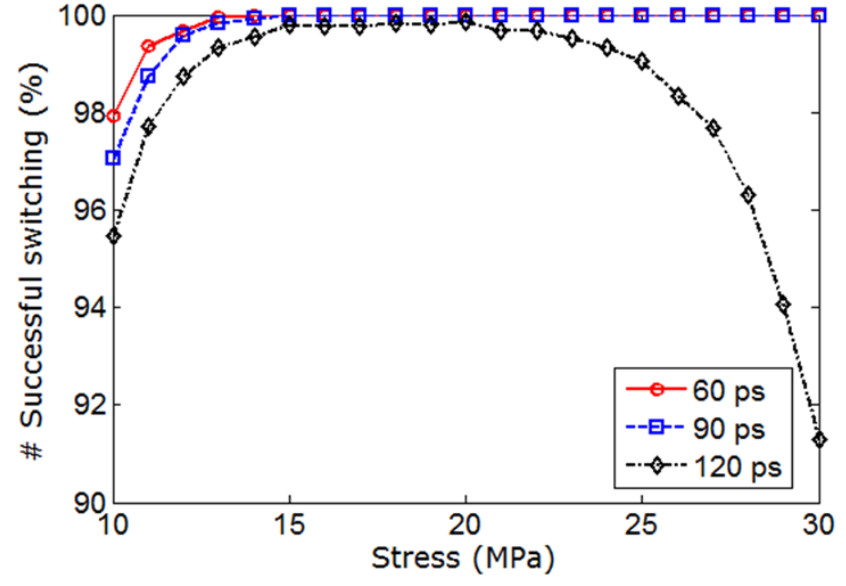

Figure 8 Percentage of successful switching events at room-temperature $(300 \mathrm{~K})$ when the magnetostrictive nanomagnet is subjected to stress between $10 \mathrm{MPa}$ and $30 \mathrm{MPa}$ with ramp duration $(60 \mathrm{ps}, 90 \mathrm{ps}$, and $120 \mathrm{ps)}$ as a parameter. The critical stress at which switching becomes $\sim 100 \%$ successful increases with ramp duration. However, at high ramp duration (e.g., 120 ps), we may not achieve $\sim 100 \%$ switching probability for any value of stress and the stress-dependence of the success probability becomes non-monotonic.

were performed for each value of stress and ramp duration to generate these results. Initial angle distributions at $300 \mathrm{~K}$ for both $\theta$ and $\phi$ are taken into account during simulations (Supplementary Fig. S2). The minimum stress needed to switch the magnetization at $0 \mathrm{~K}$ is $\sim 5 \mathrm{MPa}$, but the minimum stress needed to ensure switching at $300 \mathrm{~K}$ is $\sim 14 \mathrm{MPa}$ for $60 \mathrm{ps}$ ramp duration and $\sim 17 \mathrm{MPa}$ for 90 ps ramp duration. The minimum stress of $5 \mathrm{MPa}$ is attributed to the removal of in-plane shape anisotropy energy barrier by the stress anisotropy, while the increase of stress at $300 \mathrm{~K}$ is due to scuttling of magnetization in "bad" quadrants due to increased thermal fluctuations. When the ramp duration is longer, a higher stress is required to prevent magnetization traversing into "bad" quadrants. Hence, it is better to reduce the ramp duration (i.e., a faster ramp rate) to increase the success rate of switching at a lower stress level. Simulation results show that with 1 ps ramp duration, the critical stress reduces by $\sim 2 \mathrm{MPa}$ compared to the case of $60 \mathrm{ps}$ ramp duration.

For 120 ps ramp duration, 100\% success probability is unattainable for any value of stress since thermal agitations have higher latitude to divert the magnetization in wrong direction while stress is ramped down; at higher stresses accompanied by a long ramp duration, there occurs higher out-of-plane excursion pushing the magnetization in "bad" quadrants, which further aggravates the error probability. At very long ramp duration, the success (and error) probability becomes $50 \%$, since the magnetization would stay inplane of the magnet and during the ramp-down phase, random thermal fluctuations may equally scuttle the magnetization either in the "good" quadrants or in the "bad" quadrants.

Supplementary Figs. S4 and S5 plot the different statistical distributions of switching delay for $15 \mathrm{MPa}$ applied stress with $60 \mathrm{ps} \mathrm{ramp}$ duration. The mean and maximum switching delay as plotted in the distribution (Supplementary Fig. S5) are 0.52 ns and 1.13 ns, respectively.

\section{Discussion}

Therefore, we require the following two criteria for successful switching without creating any asymmetry in the potential landscape even in the presence of thermal fluctuations: (1) a high enough stress that keeps the magnetization more out-of-plane inside the good quadrants; and (2) a fast enough ramp rate that reduces the possibility of backtracking of magnetization while it is crossing (or even after 
crossing) the hard axis $\left(\theta=90^{\circ}\right)$ towards its destination. A high stress and a fast ramp rate also increase the switching speed and surpass the detrimental effects of thermal fluctuations.

In conclusion, we have shown that binary switching in a symmetric potential landscape is feasible, even in the presence of thermal noise. We have theoretically demonstrated such possibility in successful magnetization reversal of a single magnetostrictive particle, however, the ramification of our study is not limited to the field of nanomagnetism or to the particular shape or material used for the specimen. The out-of-plane dynamics plays the crucial role to ensure such error-resilient switching. This can open up a new methodology of binary switching since tilting the potential landscape would not be necessary and we hope that our findings would stimulate experimental research to establish the proposed methodology of binary switching.

\section{Methods}

We have solved the stochastic Landau-Lifshitz-Gilbert (LLG) equation ${ }^{29-31}$, which describes the time-evolution of magnetization vector in the presence of various torques including a torque due to random thermal fluctuations. Analytical expressions of different torques are mathematically formulated and incorporated in the stochastic LLG equation. Then the expressions of non-linear dynamics are derived analytically by solving the stochastic LLG equation in spherical coordinate system. Subsequently the expressions are numerically solved to get the simulation results. Detailed analytical steps are provided in the Supplementary Section S1.

1. Landauer, R. Irreversibility and heat generation in the computing process. IBM J. Res. Dev. 5, 183-191 (1961).

2. Keyes, R. W. \& Landauer, R. Minimal energy dissipation in logic. IBM J. Res. Dev. 14, 152-157 (1970).

3. Bennett, C. H. The thermodynamics of computation - a review. Int. J. Theor. Phys. 21, 905-940 (1982)

4. Stoner, E. C. \& Wohlfarth, E. P. A mechanism of magnetic hysteresis in heterogeneous alloys. Phil. Trans. Roy. Soc. A (London) 240, 599-642 (1948).

5. Slonczewski, J. C. Current-driven excitation of magnetic multilayers. J. Magn. Magn. Mater. 159, L1-L7 (1996).

6. Berger, L. Emission of spin waves by a magnetic multilayer traversed by a current. Phys. Rev. B 54, 9353-9358 (1996).

7. Kent, A. D., Özyilmaz, B. \& Barco, E. D. Spin-transfer-induced precessional magnetization reversal. Appl. Phys. Lett. 84, 3897 (2004).

8. Lee, O. J., Ralph, D. C. \& Buhrman, R. A. Spin-torque-driven ballistic precessional switching with 50 ps impulses. Appl. Phys. Lett. 99, 102507 (2011).

9. Stöhr, J., Siegmann, H. C., Kashuba, A. \& Gamble, S. J. Magnetization switching without charge or spin currents. Appl. Phys. Lett. 94, 072504 (2009).

10. Gamble, S. J. et al. Electric field induced magnetic anisotropy in a ferromagnet. Phys. Rev. Lett. 102, 217201 (2009).

11. Geerligs, L. J. et al. Frequency-locked turnstile device for single electrons. Phys. Rev. Lett. 64, 2691 (1990).

12. Jalil, M. B. A., Ahmed, H. \& Wagner, M. Analysis of multiple-tunnel junctions and their application to bidirectional electron pumps. J. Appl. Phys. 84, 4617-4624 (1998).

13. Spaldin, N. A. \& Fiebig, M. The renaissance of magnetoelectric multiferroics. Science 309, 391-392 (2005).

14. Eerenstein, W., Mathur, N. D. \& Scott, J. F. Multiferroic and magnetoelectric materials. Nature 442, 759-765 (2006).

15. Nan, C. W., Bichurin, M. I., Dong, S., Viehland, D. \& Srinivasan, G. Multiferroic magnetoelectric composites: Historical perspective, status, and future directions. J. Appl. Phys. 103, 031101 (2008).

16. Khitun, A., Nikonov, D. E. \& Wang, K. L. Magnetoelectric spin wave amplifier for spin wave logic circuits. J. Appl. Phys. 106, 123909 (2009).

17. Chung, T. K., Keller, S. \& Carman, G. P. Electric-field-induced reversible magnetic single-domain evolution in a magnetoelectric thin film. Appl. Phys. Lett. 94, 132501 (2009)

18. Wolf, S. A., Lu, J., Stan, M. R., Chen, E. \& Treger, D. M. The Promise of Nanomagnetics and Spintronics for Future Logic and Universal Memory. Proc. IEEE 98, 2155-2168 (2010)

19. Brintlinger, T. et al. In Situ Observation of Reversible Nanomagnetic Switching Induced by Electric Fields. Nano Lett. 10, 1219-1223 (2010).

20. Pertsev, N. A. \& Kohlstedt, H. Resistive switching via the converse magnetoelectric effect in ferromagnetic multilayers on ferroelectric substrates. Nanotechnology 21, 475202 (2010)

21. Roy, K., Bandyopadhyay, S. \& Atulasimha, J. Hybrid spintronics and straintronics: A magnetic technology for ultra low energy computing and signal processing. Appl. Phys. Lett. 99, 063108 (2011).
22. Roy, K., Bandyopadhyay, S. \& Atulasimha, J. Switching dynamics of a magnetostrictive single-domain nanomagnet subjected to stress. Phys. Rev. B 83, 224412 (2011).

23. $\mathrm{Wu}$, T. et al. Electrical control of reversible and permanent magnetization reorientation for magnetoelectric memory devices. Appl. Phys. Lett. 98, 262504 (2011).

24. Tiercelin, N. et al. Room temperature magnetoelectric memory cell using stressmediated magnetoelastic switching in nanostructured multilayers. Appl. Phys. Lett. 99, 192507 (2011)

25. Hu, J., Li, Z., Chen, L. \& Nan, C. High-density magnetoresistive random access memory operating at ultralow voltage at room temperature. Nature Commun. 2 $553(2011)$

26. Roy, K., Bandyopadhyay, S. \& Atulasimha, J. Energy dissipation and switching delay in stress-induced switching of multiferroic nanomagnets in the presence of thermal fluctuations. J. Appl. Phys. 112, 023914 (2012).

27. Kim, H. K. D. et al. Magnetoelectric Control of Superparamagnetism. Nano Lett. 13, 884-888 (2013)

28. Lei, N. et al. Strain-controlled magnetic domain wall propagation in hybrid piezoelectric/ferromagnetic structures. Nature Commun. 4, 1-7 (2013).

29. Landau, L. \& Lifshitz, E. On the theory of the dispersion of magnetic permeability in ferromagnetic bodies. Phys. Z. Sowjet. 8, 101-114 (1935).

30. Gilbert, T. L. A phenomenological theory of damping in ferromagnetic materials IEEE Trans. Magn. 40, 3443-3449 (2004).

31. Brown, W. F. Thermal fluctuations of a single-domain particle. Phys. Rev. 130, 1677-1686 (1963).

32. Cowburn, R. P., Koltsov, D. K., Adeyeye, A. O., Welland, M. E. \& Tricker, D. M. Single-domain circular nanomagnets. Phys. Rev. Lett. 83, 1042-1045 (1999).

33. Sun, J. Z. Spin-current interaction with a monodomain magnetic body: A model study. Phys. Rev. B 62, 570-578 (2000).

34. Cowburn, R. P. \& Welland, M. E. Room temperature magnetic quantum cellular automata. Science 287, 1466 (2000).

35. Beleggia, M., Graef, M. D., Millev, Y. T., Goode, D. A. \& Rowlands, G. E. Demagnetization factors for elliptic cylinders. J. Phys. D: Appl. Phys. 38, 3333-3342 (2005).

36. Chikazumi, S. Physics of Ferromagnetism (Oxford University Press Inc., New York, 1997).

37. Fidler, J. \& Schrefl, T. Micromagnetic modelling - the current state of the art. J. Phys. D: Appl. Phys. 33, R135 (2000).

38. Behin-Aein, B., Datta, D., Salahuddin, S. \& Datta, S. Proposal for an all-spin logic device with built-in memory. Nature Nanotech. 5, 266-270 (2010).

39. Brown, G., Novotny, M. A. \& Rikvold, P. A. Langevin simulation of thermally activated magnetization reversal in nanoscale pillars. Phys. Rev. B 64, 134422 (2001).

40. Abbundi, R. \& Clark, A. E. Anomalous thermal expansion and magnetostriction of single crystal $T b_{.27} D y_{.73} \mathrm{Fe}_{2}$. IEEE Trans. Magn. 13, 1519-1520 (1977).

41. Ried, K. et al. Crystallization Behaviour and Magnetic Properties of Magnetostrictive Tb-DyFe Films. Phys. Stat. Sol. (a) 167, 195-208 (1998).

42. Kellogg, R. \& Flatau, A. Experimental Investigation of Terfenol-D's Elastic Modulus. J. Intell. Mater. Sys. Struc. 19, 583-595 (2008).

43. Masys, A. J., Ren, W., Yang, G. \& Mukherjee, B. K. Piezoelectric strain in lead zirconate titante ceramics as a function of electric field, frequency, and dc bias. J. Appl. Phys. 94, 1155-1162 (2003).

44. Li, J. et al. Ultrafast polarization switching in thin-film ferroelectrics. Appl. Phys. Lett. 84, 1174-1176 (2004).

\section{Acknowledgements}

This work was supported by the U.S. National Science Foundation under Nanoelectronics for 2020 and Beyond (NEB) grant ECCS-1124714 and by the Semiconductor Research Corporation under the Nanoelectronics Research Initiative (NRI) Task 2203.001.

\section{Author contributions}

K.R. conceived the idea, performed the simulations, and wrote the paper. S.B. and J.A. commented on the manuscript. All authors discussed the results and agreed to the conclusions of the paper.

\section{Additional information}

Supplementary information accompanies this paper at http://www.nature.com/ scientificreports

Competing financial interests: The authors declare no competing financial interests

How to cite this article: Roy, K., Bandyopadhyay, S. \& Atulasimha, J. Binary switching in a 'symmetric' potential landscape. Sci. Rep. 3, 3038; DOI:10.1038/srep03038 (2013).

This work is licensed under a Creative Commons AttributionNonCommercial-NoDerivs 3.0 Unported license. To view a copy of this license visit http://creativecommons.org/licenses/by-nc-nd/3.0 